\title{
O Uso de Redes Sociais como Mecanismos para Promoção de Transparência e Participação dos Stakeholders no contexto dos Projetos
}

\author{
Ivaldir H. de Farias Junior ${ }^{1}$, Jeferson Kenedy M. Vieira ${ }^{2,3}$, Hermano P. de Moura ${ }^{2}$ \\ ${ }^{1}$ Universidade de Pernambuco (UPE) - Campus Garanhuns \\ Garanhuns, PE - Brasil \\ ${ }^{2}$ Universidade Federal de Pernambuco (UFPE) - Centro de Informática \\ Recife - PE, Brasil \\ ${ }^{3}$ Universidade Federal do Ceará (UFC) - Campus Quixadá \\ Quixadá, CE - Brasil \\ ivaldirjr@gmail.com, jefersonkenedy@ufc.br, hermano@cin.ufpe.br
}

\begin{abstract}
Organizations have increasingly sought to implement mechanisms that provide transparency and increase the participation of stakeholders in their projects. Given this scenario, this paper aims to discuss the use of social networks as instruments to promote transparency and stakeholder participation in projects. For this, the case of the Observatório Universal de Projetos is presented, a tool conceived from the narrative of a social network. In addition, research challenges related to this topic are discussed.
\end{abstract}

Resumo. As organizações têm buscado cada vez mais implementar mecanismos que proporcionem transparência e aumentem a participação dos stakeholders em seus projetos. Diante desse cenário, este trabalho tem como propósito discutir o uso de redes sociais como instrumentos de promoção da transparência e da participação dos stakeholders nos projetos. Para isso, é apresentado o caso do Observatório Universal de Projetos, uma ferramenta concebida a partir da narrativa de uma rede social. Além disso, são discutidos desafios de pesquisas relacionadas a essa temática.

\section{Introdução}

Os projetos movem os negócios para a inovação e a mudança. A única maneira de mudar uma organização, implementar uma estratégia, inovar, ou ganhar vantagem competitiva é através da execução dos projetos. Os projetos podem ser compreendidos como organizações temporárias e, por esse motivo, acabam importando alguns problemas e desafios vividos pelas organizações permanentes [Turner and Müller 2003].

Dentre esses problemas e desafios, a transparência tem ganhado destaque, como sendo uma condição e uma ferramenta necessária para os projetos e o seu gerenciamento [Betta and Boronina 2018]. A transparência pode melhorar a visão dos processos e informações organizacionais, oferecendo oportunidades de conhecimento sobre eles, reduzindo a possibilidade de omissão de informações, permitindo o controle sobre os produtos e serviços, facilitando a pesquisa e aumentando a confiança entre as organizações e a sociedade [Nunes et al. 2017]. 
O engajamento e a participação dos stakeholders também é um desafio vivenciado no contexto dos projetos. A participação dos stakeholders é fundamental para o planejamento e a execução de um projeto. Essa participação pode ser compreendida tanto como um objetivo-meio como um objetivo-fim para os projetos. Como meio, é um processo no qual os stakeholders cooperam e colaboram no projeto. Como um fim, a participação é um processo de empoderamento de novas habilidades, conhecimentos e experiência, que leva a uma maior auto-confiança e auto-gerenciamento [Kutomi and Piscopo 2013]. No contexto dos projetos públicos a participação dos stakeholders inclui a participação da sociedade nos projetos.

As organizações têm se apoiado cada vez mais em Tecnologias da Informação e Comunicação para promover transparência e participação dos stakeholders. Nesse sentido, as redes sociais podem ser utilizadas como uma ferramenta para atender a esses propósitos no contexto dos projetos [Bertot et al. 2012, Kanagarajoo et al. 2019]. Diante desse contexto, este trabalho tem o objetivo de discutir o uso das redes sociais como instrumentos para promover transparência e participação dos stakeholders nos projetos, para isso, apresentamos o caso do Observatório Universal de Projetos, uma iniciativa desenvolvida com esse propósito.

Além desta seção introdutória, este documento está organizado em mais três seções. A Seção 2 apresenta uma breve discussão sobre a fundamentação teórica relacionada as temáticas tratadas neste trabalho. Na Seção 3 é apresentado um breve relato sobre os conceitos subjacentes ao Observatório Universal de Projetos. E, finalmente, a Seção 4 apresenta as considerações finais deste estudo.

\section{Fundamentação Teórica}

Esta seção apresenta os fundamentos teóricos deste trabalho. Assim, são apresentados conceitos relacionados a: transparência e participação dos stakeholders nos projetos e o uso de redes sociais no contexto das organizações e dos projetos.

\subsection{Transparência e Participação dos Stakeholders nos Projetos}

Quando falamos sobre transparência nos projetos, as pessoas tendem a associá-la apenas aos projetos públicos com a divulgação de informações aos cidadãos [Trois et al. 2017] ou, ainda, aos projetos de desenvolvimento de software open source, com a abertura total dos artefatos dos projetos para toda a comunidade [Dabbish et al. 2013]. No entanto, é possível perceber que a necessidade de transparência está presente no dia-a-dia de todos os projetos, especialmente quando olhamos para os construtos subjacentes à transparência apresentados em [Bernstein 2012], quais sejam: monitoramento, visibilidade do processo, vigilância e divulgação; e para a seguinte categorização em pares da transparência relatadas em [Hood and Heald 2006]: transparência ascendente e descendente, transparência externa e interna, transparência de eventos e processos, transparência nominal e efetiva, transparência em retrospecto e em tempo real.

A forma como a transparência é implementada nos projetos é alterada pela dinâmica do desenvolvimento da teoria e da prática do gerenciamento de projetos - do gerenciamento clássico de projetos (representado principalmente pelo $\mathrm{PMBOK}$ ) à família de métodos iterativos-incrementais flexíveis de gerenciamento de projetos e produtos (Agile). Assim, o valor da transparência e sua operacionalização nos projetos pode variar nas diferentes abordagens de gerenciamento existentes [Betta and Boronina 2018]. 
O aumento da transparência possibilita cada vez mais a participação e o engajamento dos stakeholders nos projetos. Os stakeholders ou partes interessadas incluem todos os membros da equipe do projeto, assim como todas as entidades interessadas dentro ou fora da organização [PMI 2018]. Esses stakeholders do projeto podem incluir, mas não se limitam a: patrocinadores, clientes, usuários, fornecedores, parceiros de negócios, equipe do projetos, entre outros. As partes interessadas têm diversos níveis de responsabilidade e autoridade quando participam de um projeto. Este nível pode mudar ao longo do ciclo de vida do projeto. Seu envolvimento pode variar, desde contribuições ocasionais em pesquisas e grupos de discussão até o patrocínio total do projeto, que inclui o fornecimento de apoio financeiro, político, ou outro tipo de apoio [PMI 2018].

Dependendo dos interesses, os stakeholders podem assumir uma posição estratégica e engajada com relação ao projeto, ou, observando-se por outro lado, podem ser contrários a sua realização e, em casos extremos, até buscar a sua interrupção, contribuindo para seu fracasso [Noro 2012]. O sucesso ou fracasso de um projeto está relacionado com a percepção dos stakeholders sobre o valor criado pelo projeto e a natureza do relacionamento com a equipe do projeto. Se no passado o foco estava direcionado a dimensões como desenvolvimento e controle de escopo, cronograma e orçamento, atualmente o que se vê é a necessidade de balancear o controle e o fortalecimento dos relacionamentos dentro dos projetos, garantido, assim, o engajamento dos stakeholders, aumentando as chances de sucesso [Bourne 2006].

\subsection{Redes sociais no contexto das organizações e dos projetos}

As redes sociais estão cada vez mais inseridas no cotidiano das pessoas. O ser humano possui a necessidade de interagir com outros indivíduos, compartilhar informações, preferências, ideias, criar afinidades, apresentando assim interesses em comuns [Alméri et al. 2013]. Neste sentido, as redes sociais podem ser reais ou virtuais. As virtuais são representações computacionais de redes existentes, logo reais, ou criadas da interação de pessoas que jamais se conheceriam pessoalmente. O estudo das redes sociais não é assunto novo, mas as redes sociais passaram a ser representadas em software, o que trouxe uma nova perspectiva para o tema [Jucá 2011].

As redes sociais estão sendo utilizadas dentro das organizações para compartilhar experiências e lições aprendidas. Essas redes sociais podem ser úteis para gerentes de projeto ao colaborar com sua equipe e partes interessadas. Além dos benefícios trazidos pela comunicação ativa, uma rede social permite que os projetos aprendam uns com os outros. Isso ocorre por meio da capacidade adicional de armazenar conhecimento do projeto e, portanto, manter as informações aprendidas após o encerramento de um projeto [Furnell and Scott 2014]. Além disso, as redes sociais podem aproximar seus funcionários, clientes, fornecedores, acionistas, sociedade, ou seja, todas as partes interessadas da organização [Alméri et al. 2013].

O uso adequado das ferramentas de redes sociais apresenta diversas vantagens, dentre elas é possível destacar: oferece comunicação em tempo real, permitindo a rapidez na troca de informações; elimina distâncias geográficas de modo que possa se conectar a qualquer ambiente organizacional seja entre departamentos, áreas ou outras instituições; possui recursos para integrar reuniões online com grupos de pessoas específicas, disponibilizando maior aproveitamento de tempo e flexibilidade às possíveis urgências; permite à empresa conectar-se com o mundo profissional e se atualizar constantemente, de 
modo que acompanhe os avanços da tecnologia e mudanças comportamentais no mercado; resulta na utilização de recursos para busca e seleção de pessoas, permitindo o cadastramento de currículos via online, agrupando-os e os classificando de acordo com seus interesses definidos; possibilita ao consumidor envolver-se com as características da organização, conhecendo seu perfil, histórico, produtos e/ ou serviços, projetos e estruturas organizacionais por meio de recursos restritivos, controlados e disponibilizados pela própria empresa; quando integrado às estratégias de marketing, permite maior conhecimento relacionado aos interesses, necessidades e mudanças comportamentais dos consumidores, permitindo antecipar-se às tendências de mercado, divulgar sua marca e ampliar suas vendas [Alméri et al. 2013].

\section{O Caso do Observatório Universal de Projetos}

Um observatório pode ser definido como um instrumento, mecanismo, processo ou unidade organizacional que possibilite observar algo, proporcionando transparência a partir da coleta, consolidação, armazenamento, estudo, pesquisa, análise, compartilhamento, monitoramento e divulgação de dados, informação e conhecimento a partir de (e para) uma determinada comunidade [Vieira et al. 2020b].

O Observatório Universal de Projetos (OUP) foi idealizado para ser uma ferramenta analítica sobre o ecossistemas de projetos de qualquer natureza ao longo do tempo. Nele, os participantes deverão ser capazes de gerar e analisar grandes quantidades de dados, compartilhar o seu conhecimento, discutir e apresentar reflexões sobre os projetos existentes [Vieira et al. 2020a]. A ideia subjacente ao OUP foi concebida pelo Grupo de Pesquisa em Gestão de Projetos (GP2) do Centro de Informática (CIn) da Universidade Federal de Pernambuco (UFPE). O OUP ainda está em fase embrionária, no entanto, ele pode ser bastante útil para ilustrar o uso de redes sociais para promover transparência, participação dos stakeholders nos projetos.

O OUP foi planejado para executar quatro funções básicas: (1) obter informações de projetos; (2) validar informações de projetos; (3) disponibilizar análises de projetos; e (4) coordenar relacionamentos. A Figura 1 apresenta o fluxo de execução dessas funções. A primeira função faz referência a coleta e armazenamento dos dados e informações sobre os projetos em um repositório sob o qual o OUP opera, aqui chamado de Repositório Universal de Projetos (RUP). A segunda função prevista para o OUP é a validação das informações de projetos, nesse sentido, alguns mecanismos precisarão ser desenvolvidos para tentar garantir que as informações compartilhadas no OUP sejam confiáveis, tendo em vista que os dados sobre os projetos podem ser coletados das mais variadas fontes. Após coletados, os dados dos projetos deverão passar por processos de análises, que caracteriza a terceira função do OUP. Por fim, a quarta função do OUP, que trata da coordenação dos relacionamentos, faz referência a criação de um ambiente colaborativo que proporcione o compartilhamento de conhecimentos e experiências entre os usuários.

O OUP é apresentado como um sistema aberto baseado na narrativa de uma rede social, onde os envolvidos poderão compartilhar dados, informações e experiências sobre seus projetos [Vieira et al. 2020a]. Antes de acessar a ferramenta, os usuários devem fazer um cadastro e criar o seu perfil, assim cada usuário terá uma página na ferramenta. Os projetos são cadastrados pelos usuários, assim, cada projeto também terá uma página na ferramenta. A Figura 2 apresenta a página de um projeto no protótipo do OUP. 


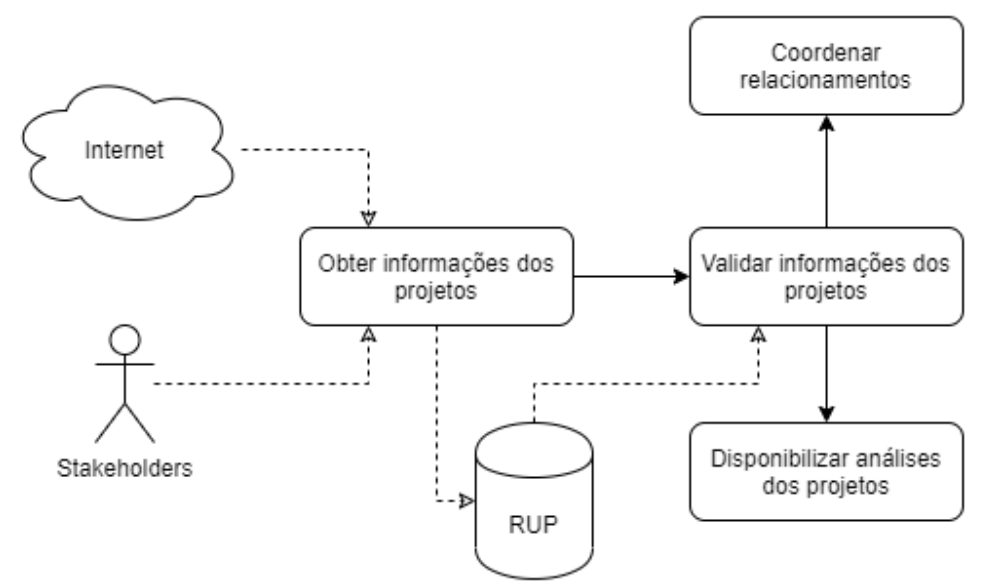

Figura 1. Fluxo operacional do OUP

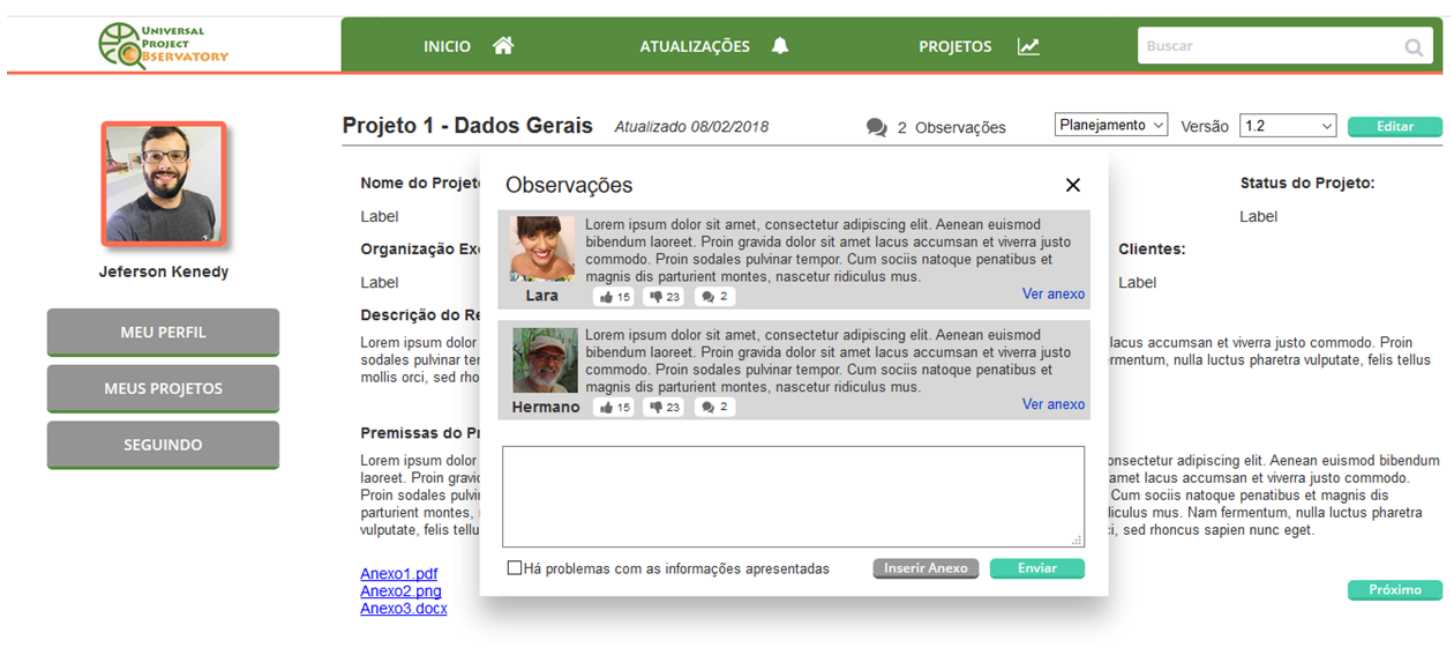

Figura 2. Página de um projeto no protótipo do OUP

Caso algum usuário queira acompanhar um projeto, recebendo atualizações sobre ele, basta "seguir" o projeto, essas atualizações chegarão no feed de cada usuário (Figura 3). Caso queira, um usuário também pode seguir uma categoria de projetos ou, ainda, seguir outros usuários para acompanhar os projetos mantidos por eles. As features like, dislike e comentar, já bastante utilizas no contexto das redes sociais, também foram implementadas no OUP, assim os usuários poderão fazer "observações" nas postagens dos projetos através de comentários, além disso, essas postagens poderão receber likes e dislikes de outros usuários, contribuindo para o engajamento da postagem.

O OUP conta ainda com ferramentas que auxiliam os usuários na construção de análises sobre os dados dos projetos, possibilitando a construção de análises que podem ser personalizadas pelos usuários (Figura ??). Também foram planejados mecanismos para denúncia de informações falsas na tentativa de evitar disseminação desse tipo de informação.

A transparência nos projetos é promovida pelo OUP na medida em que são compartilhadas informações acerca dos projetos com outros stakeholders que, usualmente, 


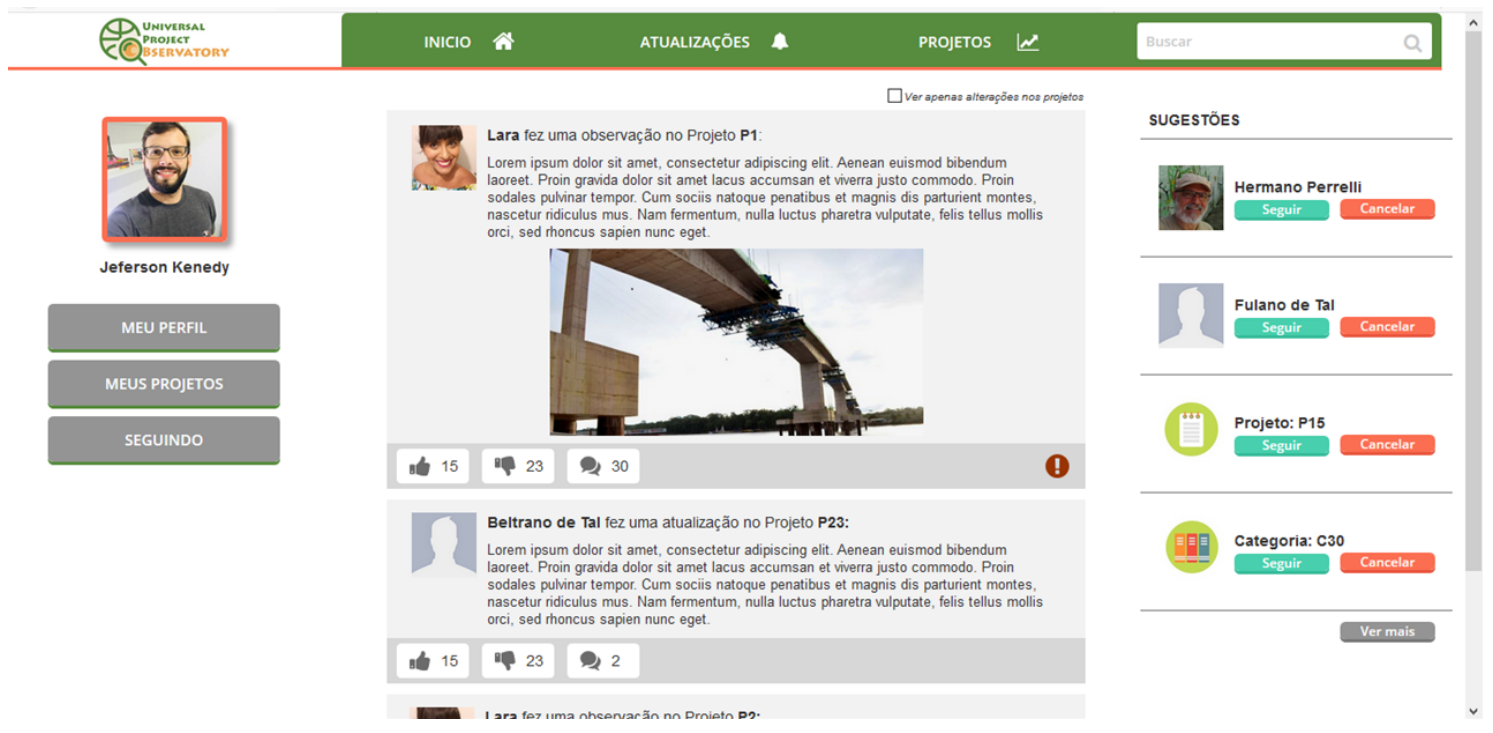

Figura 3. Página do feed de um usuário no protótipo do OUP

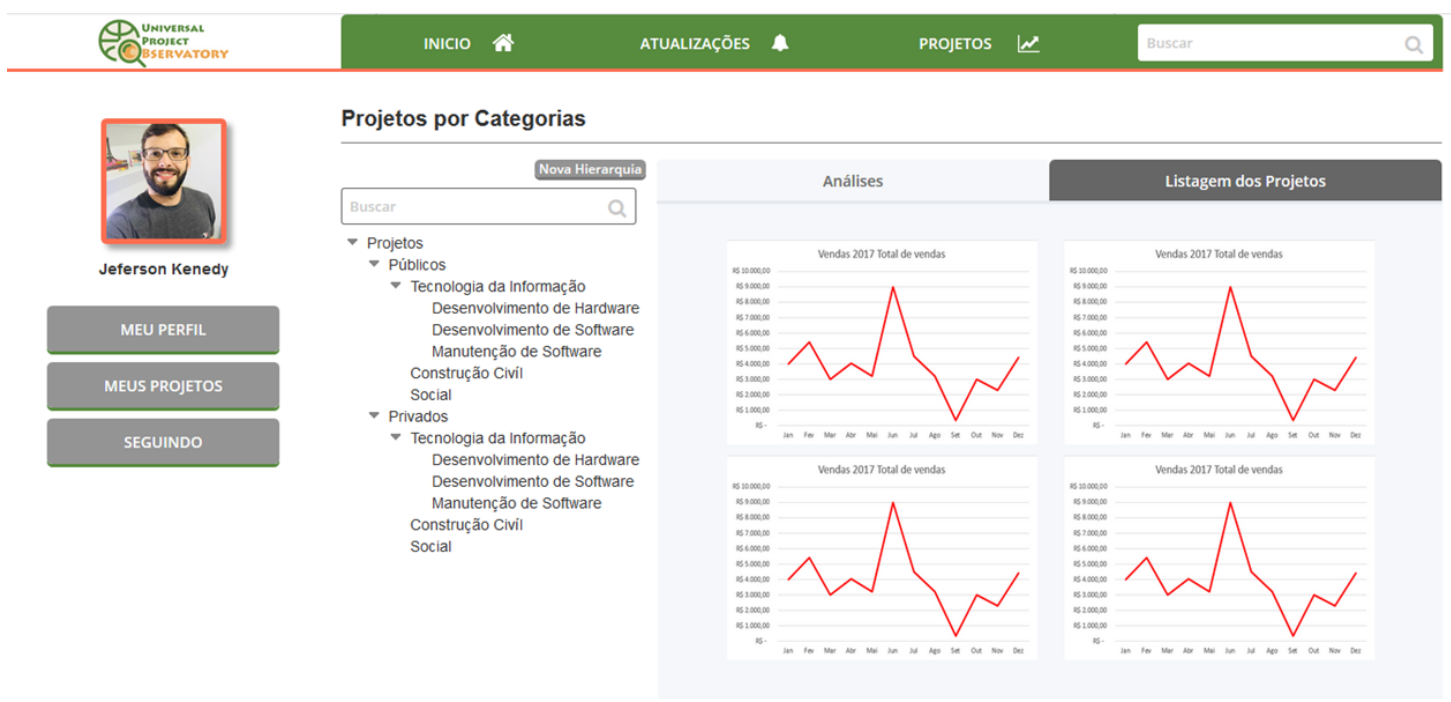

Figura 4. Página de análises dos projetos do protótipo do OUP

não teriam acesso a essas informações. Já a participação dos stakeholders nos projetos é viabilizada com a possibilidade desses stakeholders interagirem com os projetos e seus dados, possibilitando aos times e gerentes de projetos coletarem feedbacks e avaliações sobre seus projetos ainda em tempo de execução. É possível identificar no OUP muitos elementos comuns às redes sociais, esses elementos são fundamentais para que essa ferramenta cumpra o objetivo de promover transparência e a participação dos stakeholders nos projetos.

O escopo universal do OUP se apresenta como um grande desafio para a sua operacionalização. No entanto, o desenvolvimento de observatórios de projetos que implementem os recursos do OUP com escopo mais reduzido também podem ser úteis para promover transparência e participação dos stakeholders nos projetos. Em [Vieira et al. 2020a] são apresentadas iniciativas de observatórios de projetos com escopo reduzido visando experimentar os conceitos propostos pelo OUP. 


\section{Considerações Finais}

A transparência e a participação dos stakeholders tem se apresentado como desafios para as organizações e, em especial, no contexto dos projetos [Betta and Boronina 2018, Bourne 2006]. Nesta conjuntura, os recursos das redes sociais podem ser utilizadas como mecanismos na tentativa de solucionar esses desafios. Neste trabalho foi apresentado o Observatório Universal de Projetos, uma ferramenta concebida a partir da narrativa de uma rede social com o objetivo de promover transparência e aumentar a participação e o engajamento dos stakeholders nos projetos.

Diante deste contexto, esperamos que este trabalho possa trazer tanto contribuições para a academia como para o mercado. No âmbito da academia, a união dos três construtos subjacentes a esta pesquisa (transparência, participação dos stakeholders e redes sociais) no contexto dos projetos pode oferecer uma nova área de conhecimento a ser explorada com novos estudos. Já no contexto do mercado, acreditamos que podem ser concebidas, a partir dos conceitos aqui apresentados, novas ferramentas baseadas nos recursos de redes sociais, com o objetivo de proporcionar transparência e participação dos stakeholders nos projetos. O principal fator limitante deste estudo reside no fato do Observatório Universal de Projetos ainda encontrar-se em fase embrionária, o que impossibilitou uma avaliação mais precisa dos resultados que podem ser alcançados com a implementação e uso dessa ferramenta.

Este trabalho não tem o objetivo de esgotar as discussões relacionadas a esta temática, mas pode servir de ponto de partida, em especial, na identificação de desafios de pesquisa e trabalhos futuros. Dentre os desafios de pesquisa é possível destacar: a necessidade de estudos sobre aspectos tecnológicos, em especial sobre o uso e o desenvolvimento de novas tecnologias de informação e comunicação que apoiem a operacionalização das redes sociais nos projetos; e a necessidade de desenvolvimento de estudos sobre os aspectos sociais, incluindo os atores envolvidos e processos executados pelas ferramentas que implementam os recursos de redes sociais no contexto dos projetos.

\section{Referências}

Alméri, T. M., Mendes, A. d. C., Martins, L. F., and Luglio, R. G. (2013). A influência das Redes Sociais nas Organizações. Revista de Administração da FATEA, 7(7):132-146.

Bernstein, E. S. (2012). The transparency paradox: A role for privacy in organizational learning and operational control. Administrative Science Quarterly, 57(2):181-216.

Bertot, J. C., Jaeger, P. T., and Grimes, J. M. (2012). Promoting transparency and accountability through ICTs, social media, and collaborative e-government. Transforming Government: People, Process and Policy, 6(1):78-91.

Betta, J. and Boronina, L. (2018). Transparency in Project Management - from Traditional to Agile. Advances in Economics, Business and Management Research, 56(Febm):446-449.

Bourne, L. (2006). Project relationships and the stakeholder Circle. Managing, (July):117.

Dabbish, L., Stuart, C., Tsay, J., and Herbsleb, J. (2013). Leveraging transparency. IEEE Software, 30(1):37-43. 
Furnell, R. and Scott, P. (2014). Is Social Networking the future of Project Management? International Journal for Digital Society, 5(2):933-940.

Hood, C. and Heald, D. (2006). Transparency: The key to better governance? Oxford University Press.

Jucá, P. M. (2011). Uso de Redes Sociais Como Ferramenta de Estímulo à Participação na Gestão do Conhecimento dos Processos Organizacionais. PhD thesis, Universidade Federal de Pernambuco.

Kanagarajoo, M. V., Fulford, R., and Standing, C. (2019). The contribution of social media to project management. International Journal of Productivity and Performance Management, 69(4):834-872.

Kutomi, A. S. and Piscopo, M. R. (2013). Comprometimento e envolvimento dos stakeholders internos em projetos. In II Simpósio Internacional de Gestão de Projetos e Simpósio Internacional de Invoação e Sustentabilidade, pages 1-12, São Paulo.

Noro, G. D. B. (2012). A Gestão de Stakeholders em Gestão de Projetos. Revista de Gestão e Projetos, 3(1):127-158.

Nunes, V. T., Cappelli, C., and Ralha, C. G. (2017). Transparency in Information Systems. In Brazilian Computer Society (SBC), editor, I GranDSI-BR - Grand Research Challenges in Information Systems in Brazil 2016 - 2026, chapter 7, pages 73-89.

PMI (2018). A Guide to the Project Management Body of Knowledge (PMBOK Guide). Project Management Institute (PMI), $6^{\mathrm{a}}$ edition edition.

Trois, C., Weingaertner, D., Pasqualin, D., Maciel, E., Almeida, E., Silva, F., Tissot, H., Bona, L. C., Castilho, M., Didonet, M., and Sunye, M. (2017). Transparency meets management: A monitoring and evaluating tool for governmental projects. In Proceedings of IEEE/ACS International Conference on Computer Systems and Applications, AICCSA, pages 1429-1435.

Turner, J. R. and Müller, R. (2003). On the nature of the project as a temporary organization. International Journal of Project Management, 21(1):1-8.

Vieira, J. K. M., Barbosa, J. L. P., Farias Junior, I. H., and Moura, H. P. (2020a). Universal Observatory of Projects: Initial Studies. In 15th Iberian Conference on Information Systems and Technologies (CISTI), pages 1-6, Sevilla, Spain.

Vieira, J. K. M., Barbosa, J. L. P., Junior, I. H. D. F., and Moura, H. P. D. (2020b). Observatories: A Systematic Mapping of the Literature. International Journal of Development Research, 10(9):39869-39876. 


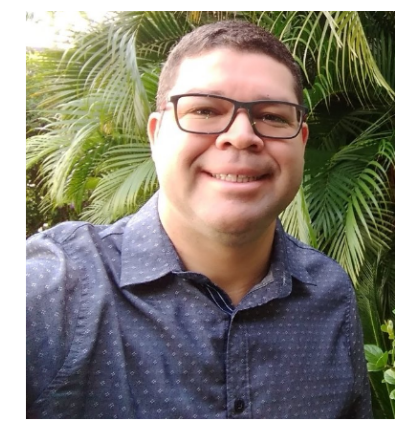

\section{Ivaldir de Farias Junior}

Graduado em Tecnologia em Processamento de Dados pela FACIR/FAPE(2004). Mestre (2008), Doutor e Pós-Doutor em Ciência da Computação no Centro de Informática da Universidade Federal de Pernambuco - UFPE. Profissional certificado em Teste de Software pelo BSTQB/ISTQB, Implementador e Avaliador MPT.BR, Implementador MPS.BR SV e Avaliador Certics, certificado ScrumMaster pela Scrum Aliance. Possui mais de 20 anos de experiência em tecnologia da informação e 13 anos atuando como professor universitário. Atualmente é professor universitário da Universidade de Pernambuco (UPE), consultor de processos e melhoria da qualidade do SOFTEX/Recife e pesquisador do grupo de pesquisa (GP2) - Project Research Group da UFPE. Executa trabalhos voluntários há mais de 7 anos. Foi Coordenador de várias ações sociais direcionadas à capacitação de mão de obra para a área de computação com foco em pessoas de baixa renda. Também, desempenha o papel de mentor profissional voluntário para alunos que querem escolher uma profissão.

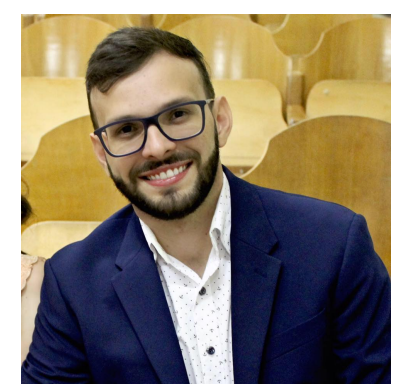

\section{Jeferson Kenedy Morais Vieira}

Bacharel em Sistemas de Informação pela Faculdade de Juazeiro do Norte - 2010. Mestre em Engenharia de Software pelo Centro de Estudos e Sistemas Avançados do Recife (CESAR School) - 2014. Tem experiência na área de Sistemas de Informação e Engenharia de Software, com ênfase em Qualidade de Software e Gestão de Projetos, atuando principalmente nos seguintes temas: Transparência Organizacional, Melhoria de Processos de Software, Modelos de Maturidade, Metodologias Ágeis e Estratégias de Melhoria de Produtividade. Atualmente é Professor Assistente na Universidade Federal do Ceará (UFC) no campus Quixadá, e está cursando o doutorado no Centro de Informática (CIn) da Universidade Federal do Pernambuco (UFPE) - desde 2018, com pesquisa direcionada ao desenvolvimento de um modelo conceitual para observatórios de projetos. 


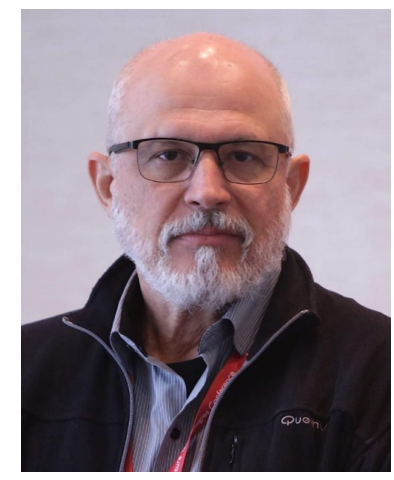

\section{Hermano Perrelli de Moura}

Atualmente é Professor Titular do Centro de Informática (CIn) da Universidade Federal de Pernambuco (UFPE). Tem pós-doutorado pela Universidade de Maryland em College Park, EUA (2011). PhD em Ciência da Computação pela Universidade de Glasgow, Escócia (1993). Mestrado em Informática pela UFPE (1989). Graduado em Engenharia Eletrônica pela UFPE (1982). É certificado PMP (2003) pelo Project Management Institute. Atua na área de Computação, com ênfase em Engenharia de Software e Sistemas de Informação, desenvolvendo pesquisas e projetos, principalmente, nas seguintes linhas: gestão de projetos, gestão de projetos de software, estudos do futuro, processo de desenvolvimento de software, gestão da TI e empreendedorismo em informática. Tendo iniciado suas atividades na área de informática em 1979, desenvolve atualmente atividades de ensino e pesquisa, a nível de graduação e pós-graduação, além de projetos de cooperação com a indústria e de gestão universitária. Foi Pró-reitor de Planejamento, Orçamento e Finanças da UFPE (2011-2015). Ocupou diversas funções administrativas no CIn como Vice-diretor, Chefe de Departamento, Coordenador de Planejamento e Finanças. Atualmente coordena o Programa Residência em Desenvolvimento de Software Emprel-UFPE e participa da coordenação do Programa CIn-Motorola. Atua nos programas de pós-graduação acadêmico e profissional em Ciência da Computação da UFPE. 